\title{
LIST OF THE PARTICIPANTS
}

\author{
Nonlinear Evolution Equations and Dynamical System \\ ICM 2002 Satellite conference at Yellow Mountains, \\ August 15-18, 2002, China
}

Chen Chunli, Department of Physics, Shanghai Jiaotong University, Shanghai, P.R. China, 200030, clchen@mail.sjtu.edu.cn

Chen Zuchi, USTC, Department of Mathematics, University of Science and Technology of China, Hefei, Anhui, China 230026, chenzc@ustc.edu.cn Chen Guiqiang, Department of Mathematics, Northwestern University, Evanston, IL 60208, USA, gqchen@math.nwu.edu

Cheng Yi, Department of Mathematics, University of Science and Technology of China, Hefei, Anhui, China 230026, chengy@ustc.edu.cn

Deng Shufeng, Department of Mathematics, Shanghai University, Shanghai, P.R. China, 200436, sfdeng@etang.com

B. Dubrovin, SISSA-ISAS, Via Beirut 2/4, 34100 Trieste, ITALY, dubrovin@sissa.it

L.D. Faddeev, Sankt-Petersburg Steklov Mathematical Institute, Laboratory of Mathematical Problems of Physics 191011, Sankt-Peterburg, Fontanka, 27, Russian, faddeev@euclid.pdmi.ras.ru

Gao Yun, Department of Mathematics and Statistics, York University, Toronto, Canada, M3J 1P3 and USTC, ygao@Yorku.ca

Gui Changfeng, Department of Mathematics, University of Connecticut, Storrs, CT 06269-3009, USA, changfenggui@yahoo.com

He Jingsong, Department of Mathematics, University of Science and Technology of China, Hefei, Anhui, China 230026, jshe@ustc.edu.cn

Hu Hengchun, Department of Physics, Shanghai Jiaotong University, Shanghai, P.R. China, 200030, hhengchun@yahoo.com.cn

Hu Sen, Department of Mathematics, University of Science and Technology of China, Hefei, Anhui, China 230026, shu@ustc.edu.cn

Hu Xing-Biao, Academia Sinica, Academy of Mathematical Sciences and Systems Sciences, Institute of Computational Mathematics and Scientific Engineering Còmputations, State Key Lab Sci and Engn Comp, Beijing, P.R. China, 100080, hxb@lsec.cc.ac.cn

Huang Yi-Zhi, Department of Mathematics, Rutgers University, 110 
Frelinghuysen Road, Piscataway, NJ 08854-8019 USA, yzhuang@math. rutgers.edu

Huang Zhenghong, Department of Computer Science, Chong Qing Technology and Business University, Chong Qing, P.R. China, hzh@ctbu.edu.cn

Ji Xiaoda, Department of Mathematics, University of Science and Technology of China, Hefei, Anhui, China 230026, jxd@ustc.edu.cn

V. Kac, Department of Mathematics, MIT, Cambridge, MA 02139 USA, kac@math.mit.edu

K. Khanin, Department of Applied Mathematics and Theoretical Physics, University of Cambridge, Cambridge, CB3 0WA, UK, kk262@newton cam.ac.uk

Jungseob Lee, Department of Mathematics, Ajou Univ., Wondcheondong, Paldal-gu, Suwon 442-749, South Korea, jslee@madang.ajou.ac.kr

D. Levi, University Roma Tre, Dipartimento Fis E Amaldi, Via Vasca Navale 84, I-00146 Rome, Italy Univ Roma Tre, Dipartimento Fis E Amaldi, I-00146 Rome, Italy, levi@amaldi.fis.uniroma3.it

Li Mengru, Department of Mathematics, Zhengzhou University, Henan, P.R. China, 450052, lmr@zzu.edu.cn

Li Ji, Department of Physics, Shanghai Jiaotong University, Shanghai, P.R. China, 200030, jhlinji@mail.jhptt.zj.cn

Li Zhen, Department of Mathematics, Zhengzhou University, Henan, P.R. China, 450052

Li Yinghua, Department of Mathematics, University of Science and Technology of China, Hefei, Anhui, China 230026, mzliyh@163.com

Li Yishen, Department of Mathematics, University of Science and Technology of China, Hefei, Anhui, China 230026, ysli@ustc.edu.cn

Liu Jin, Department of Mathematics, Shanghai University, Shanghai, P.R. China, 200436, liujinzz@163.com

Lu Yunguang, Department of Mathematics, University of Science and Technology of China, Hefei, Anhui, China 230026, yglu $2000 @ y a h o o . c o m$

Ma Hongcai, Department of Physics, Shanghai Jiaotong University, Shanghai, P.R. China, 200030, rainbowm@371.net

Peng Jiaqui, Graduate School of the Chinese Academy of Sciences, Chinese Academy of Sciences, Yuquan Road, Beijing

Tang Xiaoyan, Department of Physics, Shanghai Jiaotong University, Shanghai, P.R. China, 200030, xytang@sjtu.edu.cn

Wang Hongye, Department of Mathematics, Zhengzhou University, Henan, P.R. China, 450052, why@zzu.edu.cn

Wei Shishu, Department of Mathematics Physical Sciences Center, 
601 Elm Avenue, University of Oklahoma Norman, Oklahoma 73019 wwei@ou.edu

Xie Weiqing, Department of Mathematics and Statistics, College of Science,California State Polytechnic University, 3801 West Temple Avenue, Pomona, CA 91768,USA, wxie@csupomona.edu

Yan Qingyou, Center of Advanced Design Technology, Dalian University, Dalian, P.R. China, 116622, yanqingyou@263.net.

Ye Xiangdong, Department of Mathematics, University of Science and Technology of China, Hefei, Anhui, China 230026, yxd@ustc.edu.cn

Zhang Da-Jun, Department of Mathematics, Shanghai University, Shanghai, P.R. China, 200436, djzhang@mail.shu.edu.cn

Zhang Jingshun, Department of Mathematics, Zhengzhou University, Henan, P.R. China, 450052, zjs@zzu.edu.cn

Zhang Pu, Department of Mathematics, University of Science and Technology of China, Hefei, Anhui, China 230026, pzhang@ustc.edu.cn

Zhang Shunli, Department of Physics, Shanghai Jiaotong University, Shanghai, P.R. China, 200030, zhan $g_{s}$ hunli@situ.edu.cn, zhang $g_{s}$ hunli@hotmail.com

Zhang Yi, Department of Mathematics, Shanghai University, Shanghai, P.R. China, 200436, zh.yi@netease.com

Zhang Yufeng, School of Information Science and Engineering, Shangdong University of Science and Technology Tian, P.R. China, 271019, zhan $g_{y}$ fshandong@163.com

Zhou Zi-Xiang, Institute of Mathematics, Fudan University, Shanghai, P.R. China, 200433, zxzhou@guomai.sh.cn

Zuo Dafeng, Department of Mathematics, University of Science and Technology of China, Hefei, Anhui, China 230026, dfzuo@ustc.edu.cn

Zheng Zhong, Department of Mathematics, University of Science and Technology of China, Hefei, Anhui, China 230026, zzheng@mail.ustc.edu.cn

Zhu Meijun, Department of Mathematics Physical Sciences Center, 601 Elm Avenue University of Oklahoma Norman, Oklahoma 73019, mzhu@math.ou.edu 